\title{
Targeting diacylglycerol lipase reduces alcohol consumption in preclinical models
}

\author{
Nathan D. Winters, ${ }^{1,2,3}$ Gaurav Bedse, ${ }^{1,2}$ Anastasia A. Astafyev, ${ }^{1}$ Toni A. Patrick, ${ }^{1}$ Megan Altemus, ${ }^{1}$ Amanda J. Morgan, ${ }^{1}$ \\ Snigdha Mukerjee, ${ }^{2,3}$ Keenan D. Johnson, ${ }^{1}$ Vikrant R. Mahajan, ${ }^{1}$ Md Jashim Uddin, ${ }^{4}$ Philip J. Kingsley, ${ }^{4}$ Samuel W. Centanni, ${ }^{2,5,6}$ \\ Cody A. Siciliano, ${ }^{2,3,6}$ David C. Samuels, ${ }^{5,7}$ Lawrence J. Marnett, ${ }^{3,4}$ Danny G. Winder, ${ }^{2,5,6}$ and Sachin Patel ${ }^{1,2,3,5,6}$ \\ 1Department of Psychiatry and Behavioral Sciences, ${ }^{2}$ Vanderbilt Center for Addiction Research, ${ }^{3}$ Department of Pharmacology, ${ }^{4}$ Departments of Biochemistry and Chemistry, A.B. Hancock Jr. Memorial \\ Laboratory for Cancer Research, Vanderbilt Institute of Chemical Biology, ${ }^{5}$ Department of Molecular Physiology and Biophysics, ${ }^{6}$ Vanderbilt Brain Institute, and ${ }^{7}$ Vanderbilt Genetics Institute, Vanderbilt \\ University School of Medicine, Nashville, Tennessee, USA.
}

\begin{abstract}
Alcohol use disorder (AUD) is associated with substantial morbidity, mortality, and societal cost, and pharmacological treatment options are limited. The endogenous cannabinoid (eCB) signaling system is critically involved in reward processing, and alcohol intake is positively correlated with release of the eCB ligand 2-arachidonoylglycerol (2-AG) within the reward neurocircuitry. Here we show that genetic and pharmacological inhibition of diacylglycerol lipase (DACL), the rate-limiting enzyme in the synthesis of 2-AC, reduces alcohol consumption in a variety of preclinical mouse models, ranging from a voluntary free-access model to aversion-resistant drinking and dependence-like drinking induced via chronic intermittent ethanol vapor exposure. DAGL inhibition during either chronic alcohol consumption or protracted withdrawal did not elicit anxiogenic and depression-like behavioral effects. Last, DAGL inhibition also prevented ethanol-induced suppression of GABAergic transmission onto midbrain dopamine neurons, providing mechanistic insight into how DACL inhibition could affect alcohol reward. These data suggest that reducing 2-AG signaling via inhibition of DACL could represent an effective approach to reducing alcohol consumption across the spectrum of AUD severity.
\end{abstract}

\section{Introduction}

Alcohol use disorder (AUD) is a chronic relapsing substance abuse disorder with a lifetime prevalence of approximately $30 \%$ in the United States (1). Like disordered use of other drugs, AUD can be conceptualized as a chronic disease quintessentially characterized by lack of control over consumption-related behaviors despite adverse consequences. Patients with AUD exhibit cravings and a compulsive drive to seek and use alcohol during abstinence, and may exhibit tolerance and withdrawal symptoms, often leading to relapse (2). Societal costs related to problematic drinking led to an estimated economic burden of $\$ 249$ billion in the United States in 2010 (3) - further evidence that AUD is a critical public health concern. Current treatment approaches for AUD consist of limited pharmacological therapies combined with various forms of group and individual psychotherapy, often and most effectively in conjunction (2). Despite this, efficacy is limited, and relapse rates for AUD are high $(2,4)$, indicating the need to elucidate effective pharmacological targets for the advancement of AUD therapeutics.

Authorship note: NDW and GB contributed equally to this work.

Conflict of interest: SP is a scientific consultant for Psy Therapeutics unrelated to the present work.

Copyright: (c) 2021, American Society for Clinical Investigation.

Submitted: December 14, 2020; Accepted: July 20, 2021; Published: September 1, 2021

Reference information: J Clin Invest. 2021;131(17):e146861.

https://doi.org/10.1172/JCl146861.
A growing body of work has identified the endocannabinoid (eCB) system as a critical modulator of the effects of ethanol (EtOH) (see refs. 5, 6 for review); and the eCB system is heavily implicated in a variety of processes relevant to distinct stages of AUD, including reward processing, stress reactivity, and affect modulation (7-9), suggesting that this system may serve as a promising target for AUD therapeutic development. The eCB system is a retrograde neuromodulator system wherein the lipid-derived eCB ligands 2-arachidonoylglycerol (2-AG) and anandamide (AEA) are synthesized and released from the postsynaptic compartments of neurons and activate presynaptic cannabinoid- $1\left(\mathrm{CB}_{1}\right)$ receptors to decrease neurotransmitter release probability. EtOH mobilizes the major brain eCB 2-AG in the nucleus accumbens $(10,11)$ and in some neuronal culture models $(12,13)$. Importantly, inhibition of $\mathrm{CB}_{1}$ receptors attenuates $\mathrm{EtOH}$ self-administration in rodents $(14,15)$ and diminishes EtOH-stimulated enhancements of dopamine neuron activity in midbrain reward circuits (16), collectively suggesting that intact eCB signaling is necessary for driving alcohol-seeking behaviors.

Inhibition of $\mathrm{eCB}$ signaling is posited as a potentially effective strategy to treat $\mathrm{AUD}$, and $\mathrm{CB}_{1}$ receptor inverse agonist (rimonabant) has been explored in clinical trials for the treatment of AUD (17) as well as other addiction-related disorders $(18,19)$; but this compound was removed from the European Union market due to severe neuropsychiatric side effects (20). Given that EtOH mobilizes 2-AG in the reward circuitry in a manner correlated with EtOH intake $(10,11)$, we hypothesized that targeted inhibition of 2-AG sig- 


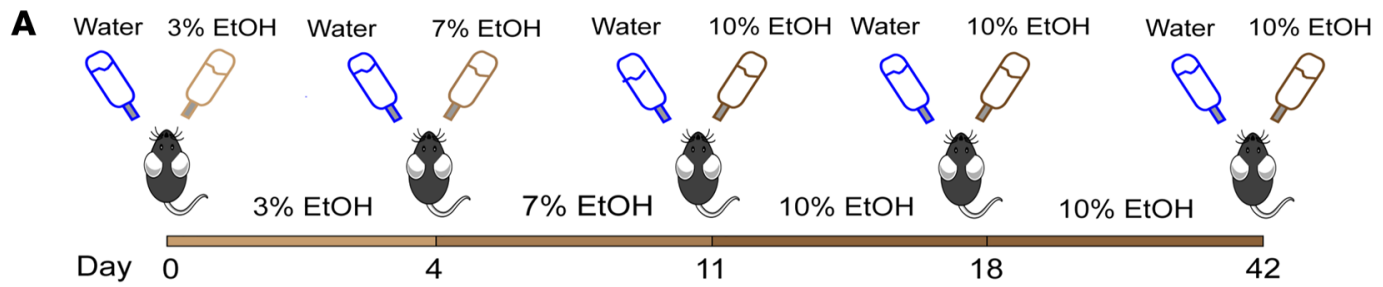

B

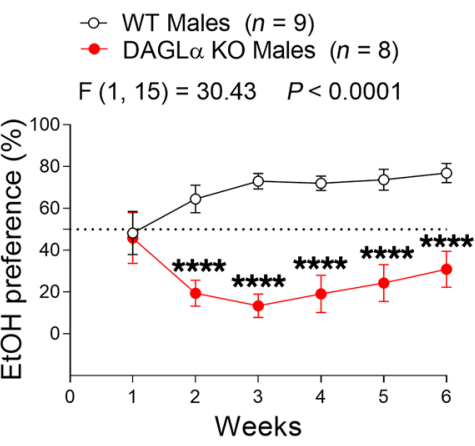

D
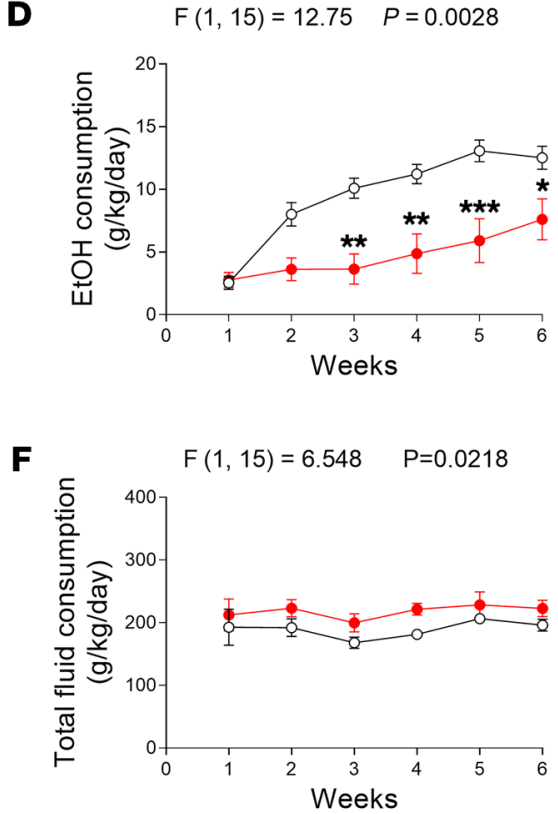

C
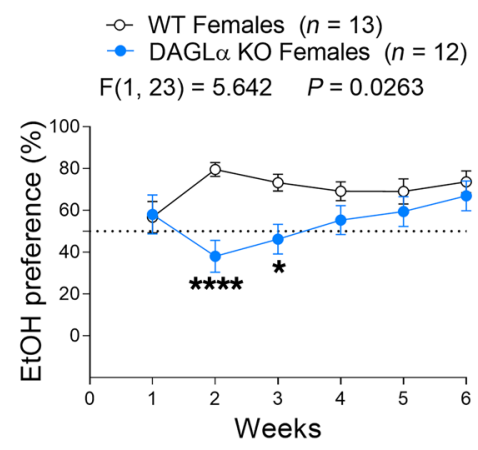

E

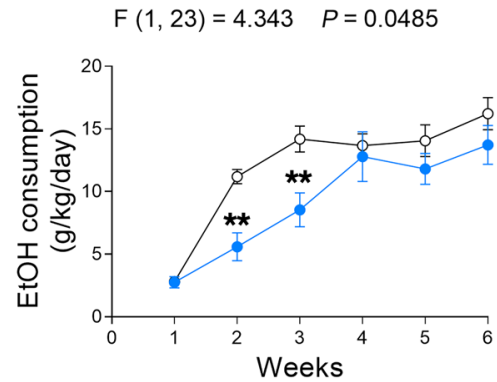

G

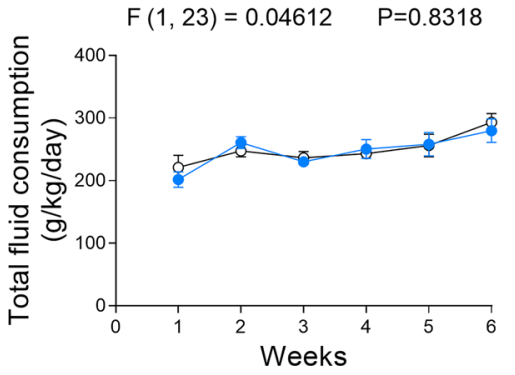

Figure 1. Genetic deletion of DAGL $\alpha$ reduces voluntary alcohol intake. (A) Schematic of the $2 \mathrm{BC}$ EtOH drinking paradigm for DACL $\alpha^{-1-}$ mice. In male and female DACL $\alpha^{-1-}$ (DAGL $\alpha \mathrm{KO}$ ) mice, (B and $\mathbf{C}$ ) EtOH preference and ( $\mathbf{D}$ and $\mathbf{E}$ ) consumption were lower than in WT mice. Male DACL $\alpha^{-1-}$ maintained lower EtOH preference (B) and consumption (D) throughout the experiment, whereas females reached WT levels in both measures by week 4 ( $\mathbf{C}$ and $\mathbf{E}$ ). ( $\mathbf{F}$ and $\mathbf{G}$ ) Male and female DACL $\alpha^{-/-}$mice showed no difference in total fluid consumption. Data analyzed by repeated-measure 2-way ANOVA, followed by a Holm-Šidák test for multiple comparisons between genotypes. Sample size $n, P$, and $F$ values for main effects of genotype, and significance for post hoc multiple comparisons are reported in the graphs. ${ }^{*} P<0.05,{ }^{* *} P<0.01,{ }^{* * *} P<$ $0.001,{ }^{* * * *} P<0.0001$. Data are presented as mean $\pm \mathrm{SEM}$. naling (which would thereby leave $\mathrm{AEA} / \mathrm{CB}_{1}$ signaling intact) could be an effective approach to reducing EtOH drinking with a reduced risk for adverse effects. Here we implemented genetic and pharmacological strategies to test the hypothesis that inhibiting diacylglycerol lipase (DAGL), the rate-limiting enzyme in 2-AG synthesis, reduces EtOH drinking behaviors without facilitating the development of anxiety- and depression-like behaviors after chronic EtOH drinking. We found that genetic deletion of DAGL $\alpha$, the primary DAGL isoform in the adult brain (21), or treatment with the pharmacological DAGL inhibitor DO34 (22) decreased EtOH consumption across a range of clinically relevant models; and did not exacerbate negative affective behaviors in chronically drinking mice or during protracted abstinence. Collectively, our data suggest that DAGL inhibition could be a promising and broadly applicable therapeutic strategy to reduce alcohol drinking across the spectrum of AUD.

\section{Results}

Genetic and pharmacological inhibition of DAGL decreases voluntary EtOH drinking. Having previously established that genetic deletion of DAGL $\alpha$ results in reduced brain 2-AG levels $(9,23)$, we evaluated the effects of DAGL $\alpha$ deletion on EtOH drinking behavior. Separate cohorts of male and female DAGL $\alpha^{-/-}$mice and WT littermates were exposed to a continuous-access 2-bottle-choice (2BC) EtOH drinking paradigm (Figure 1A). Both male and female DAGL $\alpha^{-/-}$ mice showed lower EtOH preference (Figure 1, B and C) and consumption (Figure 1, D and E) relative to their WT littermates. Of note, DAGL $\alpha^{-/-}$males maintained lower preference and consumption for the duration of the experiment (Figure 1, B and D), whereas female DAGL $\alpha^{-1-}$ mice reached WT levels by week 4 (Figure 1, $\mathrm{C}$ and $\mathrm{E})$. DAGL $\alpha^{-1-}$ mice showed no difference in total fluid consumption relative to WT controls (Figure 1, F and G). We next eval- 
uated the effects of pharmacological DAGL inhibition on alcohol drinking by using the DAGL inhibitor DO34 (22). Previously, we demonstrated that DO34 $(50 \mathrm{mg} / \mathrm{kg})$ significantly decreases brain 2-AG levels 2 hours after administration (9). Here, separate cohorts of male and female $\mathrm{C} 57 \mathrm{BL} / 6 \mathrm{~J}$ mice were exposed to the $2 \mathrm{BC}$ EtOH drinking paradigm. After 6 weeks of stable drinking, DO34 $(50 \mathrm{mg} / \mathrm{kg}$ ) was administered for 3 consecutive days (Figure $2 \mathrm{~A}$ ). DO34 reduced EtOH preference nonsignificantly in males and significantly in females (Figure 2, B and C); and significantly reduced consumption in mice of both sexes (Figure 2, D and E). DO34 did not significantly alter total fluid consumption by mice of either sex at any time point (Figure 2, F and G). Vehicle control injections had no effect on EtOH preference (Supplemental Figure 2, A and $\mathrm{D}$; supplemental material available online with this article; https:// doi.org/10.1172/JCI146861DS1) or consumption (Supplemental Figure 2, B and E). DO34 had no effect on body weight in female mice (Supplemental Figure 2F) but caused a small but significant decrease in body weight in males that reversed upon cessation of drug treatment (Supplemental Figure 2C). Due to their higher EtOH preference and consumption levels, female C57BL/6J mice were used for all drinking and behavioral experiments thereafter.

Although our convergent genetic and pharmacological studies indicated a role for DAGL in alcohol consumption, DO34 does exhibit some activity at other targets, such as $\alpha / \beta$-hydrolase domain-containing 2 and 6 (ABHD2/6), platelet-activating factor acetylhydrolase 2 (PAFAH2), carboxylesterase 1C (CES1C), and phospholipase $A_{2}$ group 7 (PLA2G7; ref. 22). To confirm that the DO34 effects on EtOH consumption and preference were specific to DAGL inhibition, we utilized the structural analog DO53, which retains the off-target activity profile of DO34 but does not inhibit DAGL (22). We administered DO53 $(50 \mathrm{mg} / \mathrm{kg})$ to a cohort of female mice stably drinking $10 \% \mathrm{EtOH}$ on our $2 \mathrm{BC}$ paradigm for 5 consecutive days. EtOH consumption was transiently reduced by DO53 treatment (Figure 2I); however, this was paralleled by a nonspecific decrease in total fluid consumption (Figure 2J). Accordingly, there was no effect on EtOH preference (Figure 2H) and no change in body weight (Supplemental Figure 3) during the 5-day DO53 treatment. These effects are in contrast to the persistent decrease in $\mathrm{EtOH}$ preference and consumption (Figure 2, B-E) and unchanged fluid consumption (Figure 2, F and $\mathrm{G})$ observed during DO34 treatment. Combined with our genetic studies using DAGL $\alpha^{-/-}$mice, these control data suggest that the effects of DO34 were mediated through DAGL inhibition, rather than off-target mechanisms.

DO34 depletes brain 2-AG levels similarly in naive and chronically drinking mice and does not affect blood EtOH concentrations. Given that our results demonstrating DO34-mediated reductions in EtOH consumption were from mice that had been chronically drinking, we next verified that DO34 causes comparable reductions in brain 2-AG levels after prolonged alcohol exposure. To do this, we collected brains from naive or chronically (6 weeks, 2BC $20 \% \mathrm{EtOH}$ ) drinking mice after 4 days of repeated DO34 injections $(50 \mathrm{mg} / \mathrm{kg})$. Brains were collected 2 hours after the final DO34 injection and hemisected, and half brain samples were prepared for mass spectrometric analysis of bulk tissue levels of endocannabinoids and related lipid metabolites. As expected, DO34 caused a strong depletion of 2-AG (Figure $2 \mathrm{~K}$ ) and its major metabolite, arachidonic acid (AA; Figure 2L) in all mice, regardless of alcohol history. Levels of prostaglandins E2 (PGE2) and D2 (PGD2), which are derived downstream from AA (24), were also decreased in DO34-treated mice of both groups (Figure 2, M and $\mathrm{N})$. Furthermore, there was no significant effect on AEA levels in either group (Figure 2O).

A possible indirect mechanism we sought to rule out is potential pharmacokinetic interaction between DO34 and blood EtOH concentration (BEC). One study reported higher BECs in $\mathrm{CB}_{1}$ receptor-knockout mice after i.p. EtOH injection, albeit only at a high dose that exceeded clinically relevant BECs (25). To address this, we injected a cohort of EtOH-naive mice with either vehicle or DO34 $(50 \mathrm{mg} / \mathrm{kg})$ for 3 consecutive days, and 2 hours after the last injection delivered an i.p. bolus of EtOH $(3 \mathrm{~g} / \mathrm{kg})$. Thirty minutes after EtOH injection, trunk blood was collected and BEC measurements were taken. We chose this method because the 30-minute time point captures peak BEC, according to previously reported pharmacokinetic data (26), and inherently reduces variability in EtOH exposure relative to voluntary drinking sessions. Repeated DO34 treatment had no effect on BEC at the 30-minute time point (Supplemental Figure 4), suggesting that DO34's effects on alcohol drinking were not due to pharmacokinetic interactions leading to altered BECs or subsequent degrees of intoxication.

Effects of 2-AG modulation are specific to EtOH and not bidirectional. To confirm that the effects of DO34 were specific to EtOH and not generalized reward seeking, we tested the effect of DO34 in a $2 \mathrm{BC}$ sucrose preference paradigm. DO34 had no effect on sucrose preference, suggesting a degree of specificity for EtOH over natural rewards (Supplemental Figure 5A). Furthermore, given that depletion of 2-AG reduces EtOH drinking, we next tested whether augmentation of 2-AG would increase $\mathrm{EtOH}$ drinking. The monoacylglycerol lipase inhibitor JZL-184 (10 mg/kg) was administered for 3 consecutive days to mice drinking 10\% EtOH in the 2BC drinking paradigm. JZL-184 had no significant effect on EtOH preference or consumption (Supplemental Figure 5 , B and C) and did not affect body weight (Supplemental Figure 5D), indicating that the effects of 2-AG modulation on EtOH drinking were not bidirectional.

Pharmacological DAGL inhibition reduces aversion-resistant EtOH drinking. Alcohol seeking despite negative consequences is recognized as a key element of AUD and is a major obstacle to effective AUD treatment $(2,27)$. To determine whether DAGL inhibition could reduce EtOH consumption in a more clinically relevant model, we utilized an aversion-resistant drinking model wherein animals actively consume EtOH that has been adulterated with the bitter tastant quinine (27). First, we validated this model by quinine adulteration in one of the 2 bottles after 4 weeks of the $2 \mathrm{BC}$ paradigm and determined the preference for and consumption of the quinine-adulterated bottles. Bottles of water, $10 \% \mathrm{EtOH}$, and $20 \% \mathrm{EtOH}$ were adulterated with quinine at 0.01, 0.03, and 0.1 $\mathrm{g} / \mathrm{L}$. Mice receiving water and quinine (water + quinine; 0.03 and $0.1 \mathrm{~g} / \mathrm{L}$ ) exhibited low preference (<7\%; Supplemental Figure $6 \mathrm{~A})$; however, those receiving $10 \%$ and $20 \% \mathrm{EtOH}+$ quinine maintained a higher preference relative to the water + quinine condition. Mice drinking 20\% EtOH + quinine showed higher EtOH consumption compared with mice drinking $10 \% \mathrm{EtOH}+$ quinine (Supplemental Figure 6B), leading us to use $20 \% \mathrm{EtOH}$ for examining the effects of 
A

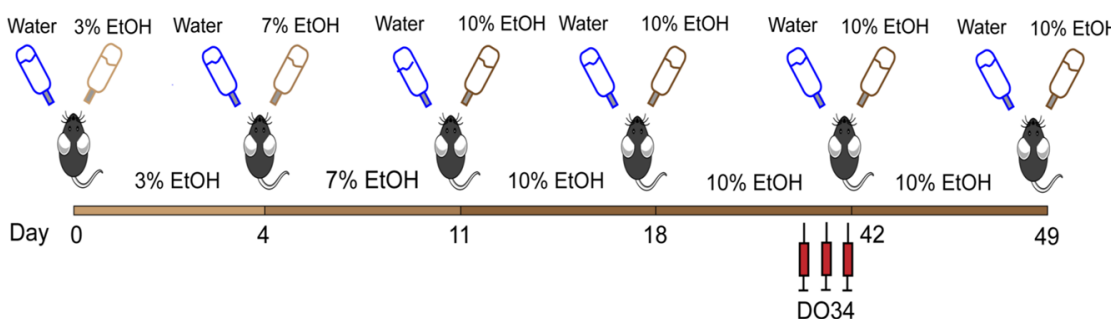

B

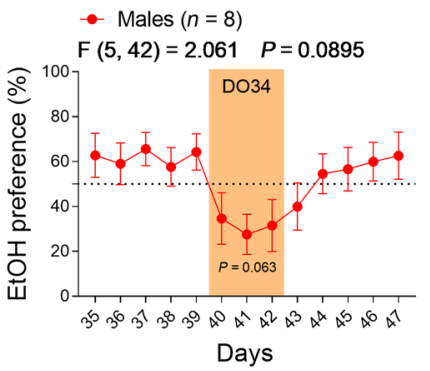

D
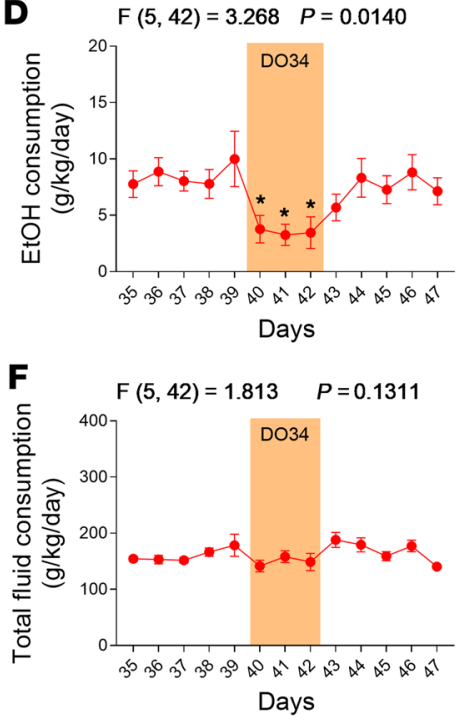

C

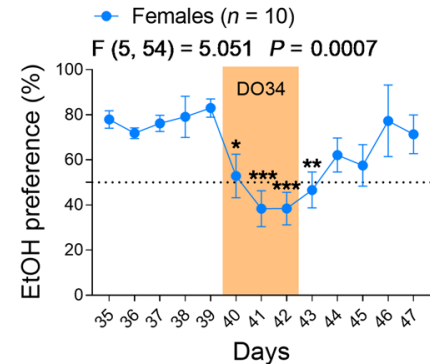

E

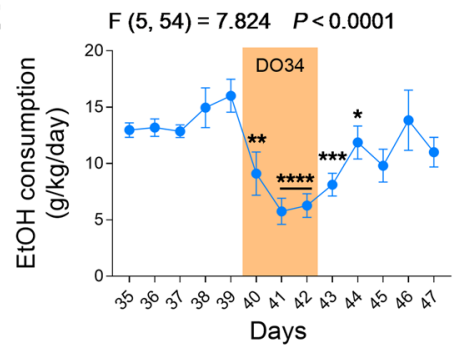

G

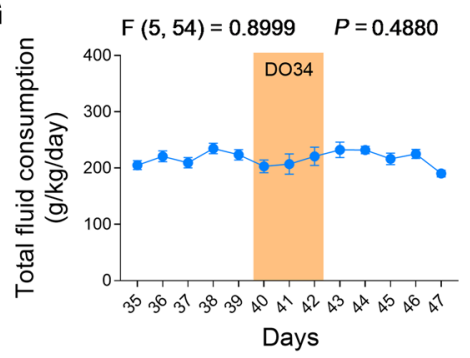

DO53 = inactive analog

H

Females $(n=15)$

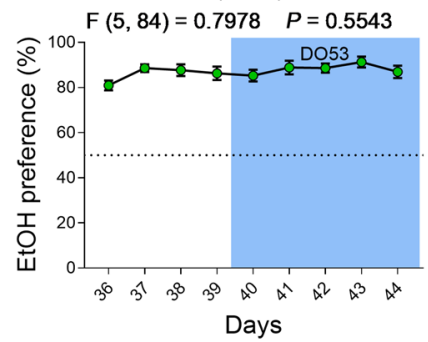

I

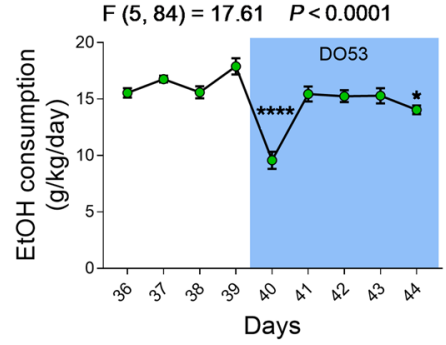

J

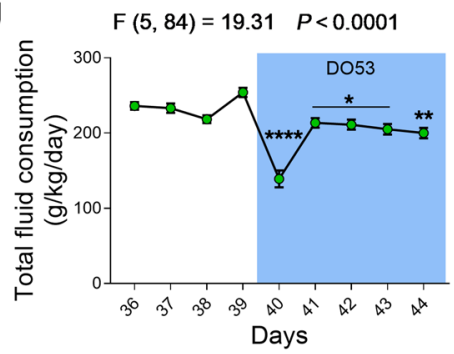

K

2-AG

$F(1,36)=379.5$

$P<0.0001$

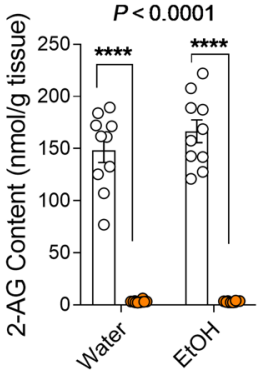

$\mathbf{L}$

$F(1,36)=1207$

$P<0.0001$

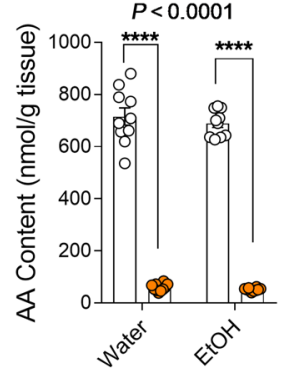

M PGE2 $F(1,36)=16.62$

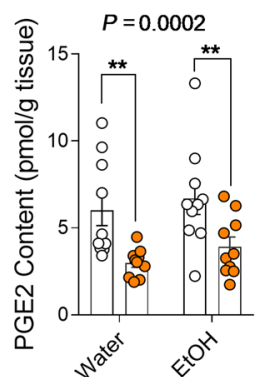

$\mathbf{N}$

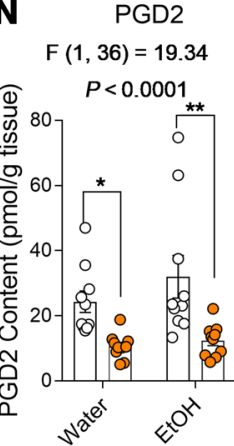

- $A E A$ $F(1,36)=5.52$

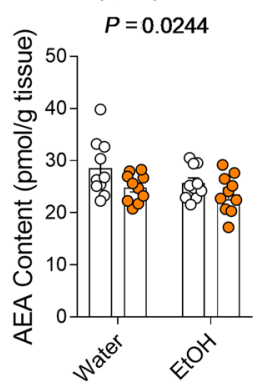

Vehicle

(Water, $n=10$ EtOH, $n=10$ )

DO34

- (Water, $n=10$ $\mathrm{EtOH}, n=10$ )

Figure 2. Pharmacological inhibition of DAGL by D034 reduces voluntary alcohol intake and produces similar brain lipid profiles in naive and chronically drinking C57BL/6) mice. (A) Schematic of 2BC EtOH drinking paradigm for mice receiving D034 treatment. (B) DO34 (3 days) nonsignificantly reduced EtOH preference in male mice and $(\mathbf{C})$ significantly reduced EtOH preference in female mice. ( $\mathbf{D}$ and $\mathbf{E})$ DO34 reduced EtOH consumption in both $(\mathbf{D})$ male and $(\mathbf{E})$ female mice. $(\mathbf{F}$ and G) DO34 had no effect on total fluid consumption in (F) male or (C) female WT mice. (H) The analog compound DO53 (5 days) had no effect on EtOH preference in female mice. (I) DO53 reduced EtOH consumption in female mice, and this was paralleled by (J) a nonspecific reduction in total fluid consumption. (K-0) DO34 (4 days) depleted brain (K) 2-AG, (L) AA, (M) PGE2, and (N) PGD2 levels similarly in naive and chronically (6 weeks, 20\% EtOH) drinking mice, with (0) no effect on AEA levels. B-G: Data analyzed by 1-way ANOVA for time points 39-44, followed by a Holm-Šidák test for multiple comparisons to baseline (day 39) control. H-J: Data analyzed by 1-way ANOVA for averaged baseline (days 36-39) and individual treatment days, followed by a Holm-Šidák test for multiple comparisons to averaged baseline. K-0: Data analyzed by 2-way ANOVA, followed by a Holm-Šidák test for multiple comparisons between vehicle and D034. All D034 and D053 treatments were dosed at $50 \mathrm{mg} / \mathrm{kg}$. Sample size $n, P$, and $F$ values for main effects of drug treatment and significance for post hoc multiple comparisons are reported in the graphs. ${ }^{*} P<0.05,{ }^{* *} P<0.01,{ }^{* * *} P<0.001,{ }^{* * *} P<0.0001$. Data are presented as mean \pm SEM. 
Aversion-Resistant Drinking

A

- Water $(n=8)$

- $20 \%$ EtOH $(n=10)$

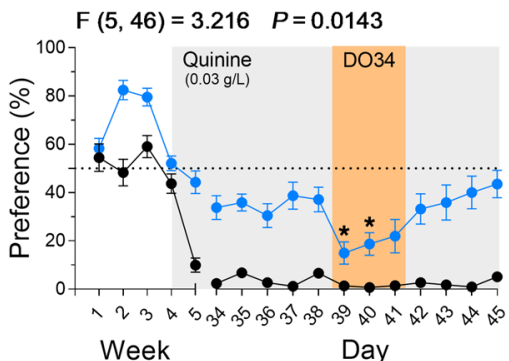

B

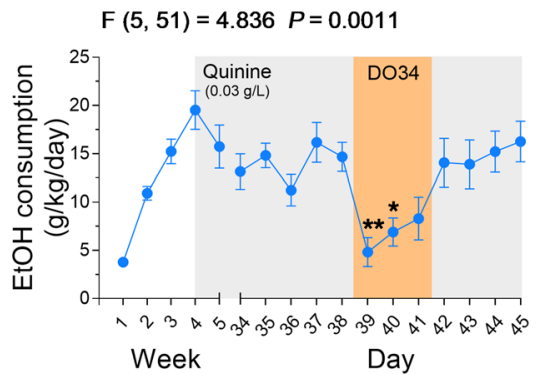

C

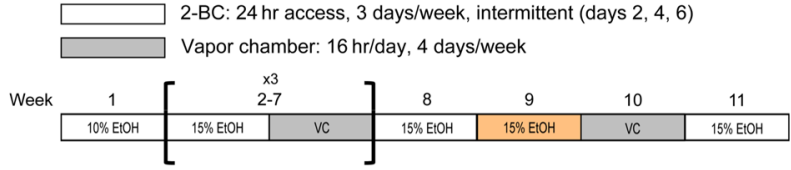

D

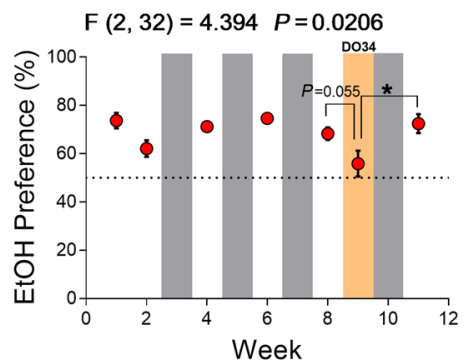

$\mathbf{F}$

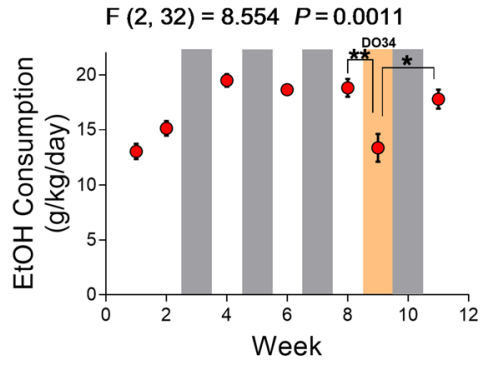

E

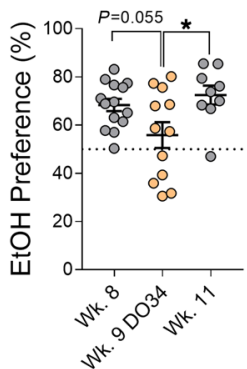

G

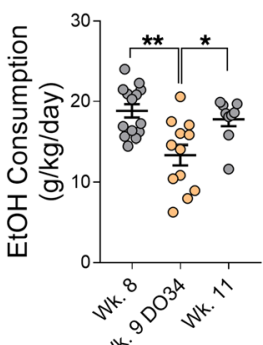

Chronic Drinking Behavior

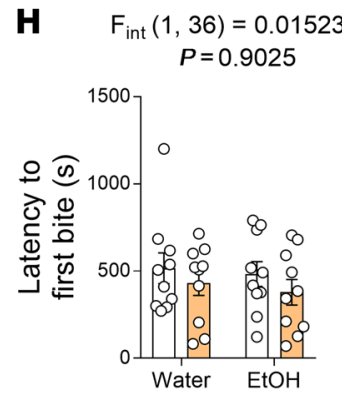

NIFS
I $\quad F_{\text {int }}(1,36)=0.244$

$P=0.6234$

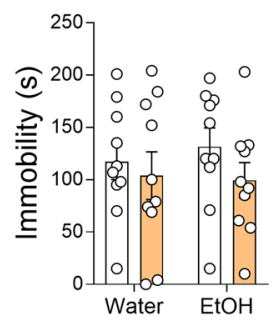

FST
J $F_{\text {int }}(1,36)=0.004763$

$P=0.9454$

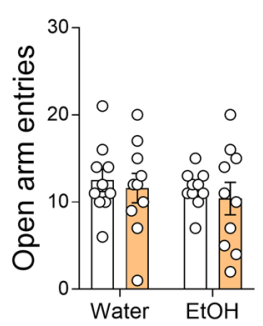

Vehicle

(Water, $n=10$

$\mathrm{EtOH}, n=10$ )

DO34

(Water, $n=10$

EtOH, $n=10$ )

Figure 3. D034 decreases EtOH intake across aversion-resistant and chronic intermittent models of EtOH drinking and does not precipitate negative affective phenotypes after chronic EtOH drinking. (A) DO34 (3 days) decreased preference and (B) consumption of $20 \% \mathrm{EtOH}+0.03 \mathrm{~g} / \mathrm{L}$ quinine (blue). Water + quinine control is shown for reference (black). (C) Schematic depicting the CIE EtOH exposure paradigm. (D and E) D034 (6 days) caused a nonsignificant reduction in $\mathrm{EtOH}$ preference $(P=0.055)$ and $(\mathbf{F}$ and $\mathbf{G})$ significantly decreased consumption of $15 \%$ EtOH in the $\mathrm{CIE}$ model. $\mathbf{E}$ and $\mathbf{G}$ show individual mouse EtOH preference and consumption values from $\mathbf{D}$ and $\mathbf{F}$, respectively. (H-K) DO34 had no effect on (H) latency to first bite in the NIFS test, (I) immobility time in the FST, or (J) open-arm entries in the EPM in water control or chronically (6 weeks, $2 \mathrm{BC}, 20 \%$ EtOH) drinking mice. (K) DO34 treatment mildly increased immobility time in the EPM in EtOH-drinking mice. A and B: Data were analyzed by 1-way ANOVA for time points 38-43, followed by a Holm-Šidák test for multiple comparisons to baseline (day 38). D-G: Data analyzed by 1-way ANOVA on weeks 8, 9, and 11, followed by a Holm-Šidák test for multiple comparisons between these 3 time points. H-K: Data analyzed by 2-way ANOVA, followed by a Holm-Šidák test for multiple comparisons between all groups. Female C57BL/6] mice were used in all experiments, and D034 was dosed at $50 \mathrm{mg} / \mathrm{kg}$. Sample size $n$ is reported in graphs for experiments in $\mathbf{A}$ and $\mathbf{B}$ and $\mathbf{H}-\mathbf{K} . \mathbf{n}=$ 9-14 mice in $\mathbf{D}-\mathbf{G} . P$ and $F$ values for main effects of $(\mathbf{A}-\mathbf{G})$ drug treatment or $(\mathbf{H}-\mathbf{K})$ EtOH $\times$ DO34 interaction and significance for post hoc multiple comparisons are reported in graphs $\left({ }^{*} P<0.05,{ }^{* *} P<0.01\right)$. Data are presented as mean \pm SEM.

DO34 on aversion-resistant drinking. We selected $0.03 \mathrm{~g} / \mathrm{L}$ quinine for further experiments, as mice exhibited robust aversion-resistant drinking at this concentration (Supplemental Figure 6A).

To test the effects of DO34 in this model, we used a separate cohort of $2 \mathrm{BC}$ mice and added $0.03 \mathrm{~g} / \mathrm{L}$ quinine to bottles of either water (control mice) or $20 \% \mathrm{EtOH}$ after 4 weeks of stable $2 \mathrm{BC}$ drinking. Mice receiving $20 \% \mathrm{EtOH}+$ quinine showed a higher pref- erence compared with the water + quinine mice (Figure 3A). DO34 treatment $(50 \mathrm{mg} / \mathrm{kg}, 3$ days) significantly reduced the EtOH preference (Figure 3A) and consumption (Figure 3B), although results on day 3 of treatment failed to reach statistical significance. DO34 treatment had no effect on total fluid consumption (Supplemental Figure $6 \mathrm{C}$ ). These data suggest that DAGL inhibition can reduce aversion-resistant alcohol consumption. 

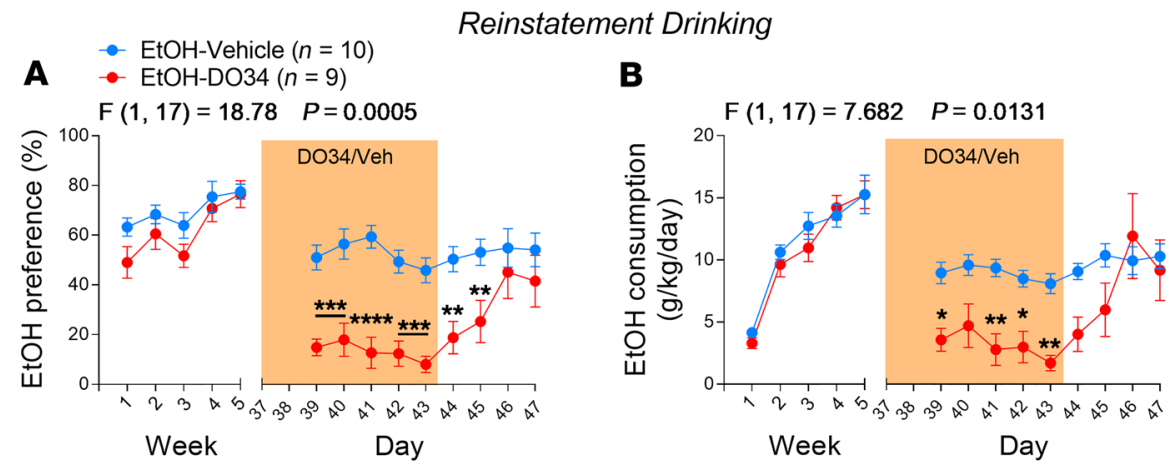

\section{C}

Withdrawal Behavior
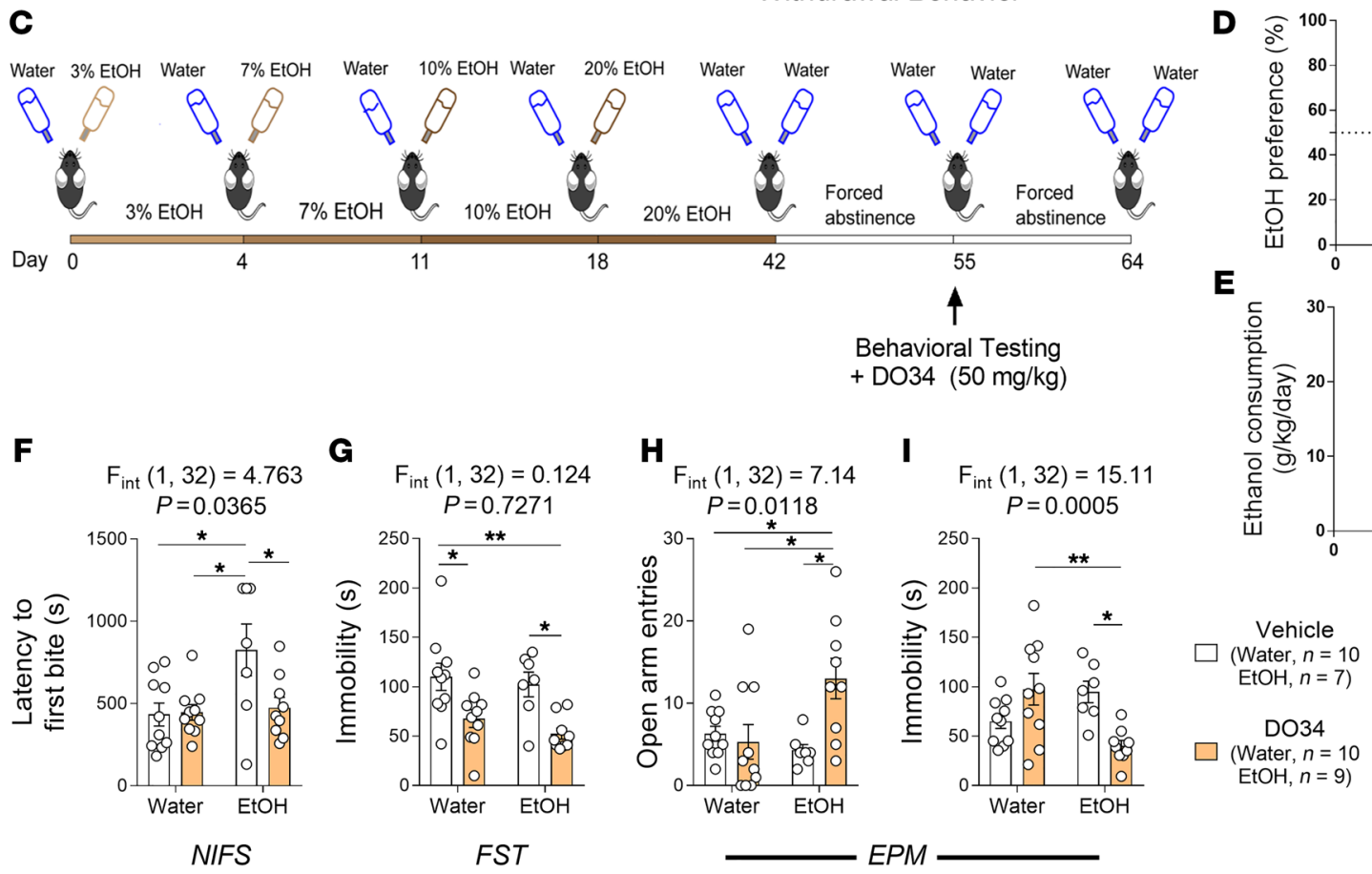

Figure 4. DO34 reduces reinstatement of EtOH drinking and does not precipitate negative affective phenotypes during protracted abstinence. (A) D034 (7 days) reduced preference and (B) consumption relative to vehicle upon reinstatement of $10 \%$ EtOH drinking after 5 weeks of drinking and 10 days of withdrawal. DO34 treatment was initiated 2 days before onset of reinstatement of EtOH access. (C) Schematic depicting $2 \mathrm{BC}$ drinking paradigm for behavioral testing in withdrawal. (D) EtOH preference and (E) consumption in mice tested in the withdrawal cohort. (F) EtOH-withdrawn mice showed a higher latency to first bite in the NIFS test, which was reduced to baseline with D034 treatment. (C) D034 treatment decreased immobility time in the FST in both groups. (H and I) DO34 treatment $\mathbf{( H )}$ increased the number of open-arm entries and (I) decreased immobility time in the EPM only in EtOH-withdrawn mice. A and B: Data analyzed by repeated-measure 2-way ANOVA, followed by a Holm-Šidák test for multiple comparisons between treatment conditions. F-I: Data were analyzed by 2-way ANOVA, followed by a Holm-Šidák test for multiple comparisons between all groups. D034 was dosed at 50 $\mathrm{mg} / \mathrm{kg}$. Sample size $n, P$, and $F$ values for main effects of (A and $\mathbf{B})$ drug treatment or $(\mathbf{F}-\mathbf{I})$ EtOH $\times$ DO34 interaction, and significance for post hoc multiple comparisons are reported in graphs. ${ }^{*} P<0.05,{ }^{* *} P<0.01,{ }^{* *} P<0.001,{ }^{* * *} P<0.0001$. Data are reported as mean \pm SEM.

Efficacy of DAGL inhibition is maintained following chronic intermittent EtOH exposure and dependence-like drinking. AUD exists on a severity spectrum and involves a shift from problematic alcohol use to dependence $(2,28)$. This transition is driven by a shift from positive to negative reinforcement (28) and thus may involve differing neurobiological mechanisms in early versus severe, late stages of AUD. Critical to the development of pharmacotherapies for AUD is the examination of relative efficacy during various stages of AUD. To address this, we examined the efficacy of DO34 in mitigating alcohol consumption in mice that had been subjected to a model of chronic intermittent EtOH (CIE) exposure to develop escalation and dependence-like drinking. CIE models are widely used to develop $\mathrm{EtOH}$ dependence in rodents and generally result in more severe AUD symptomatology and higher intoxication levels relative to continuous-access models (27, 29, 30 ). Mice were exposed to intermittent 2BC $15 \%$ EtOH drinking for 24-hour periods 3 times per week on alternating days. In alternating weeks, mice were exposed to EtOH vapor inhalation 16 hours per day, 4 times per week (Figure 3C). Mice showed a clear escalation in $\mathrm{EtOH}$ preference and consumption following the first week of EtOH vapor exposure. Following $32 \mathrm{BC}$-vapor chamber cycles and 1 additional week of baseline drinking, mice were treat- 
A

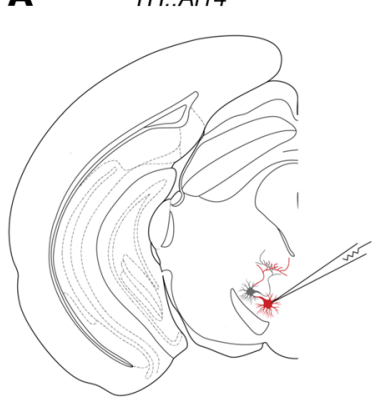

B $\quad F_{\text {int }}(1,34)=3.603 \quad P=0.0662$

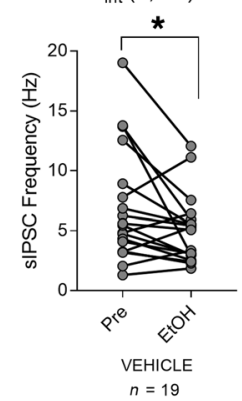

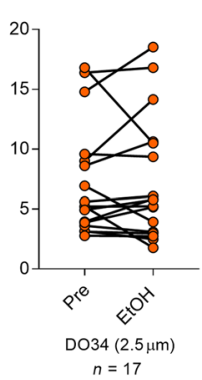

Veh
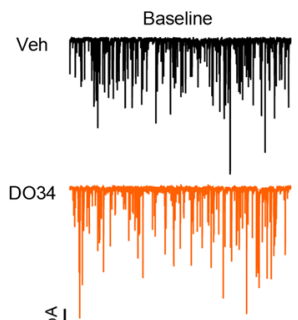
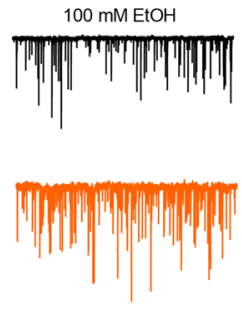

C

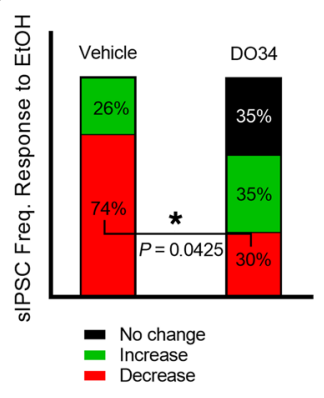

Figure 5. D034 prevents EtOH suppression of posterior VTA GABA transmission. (A) Schematic depicting recording strategy used to identify putative dopamine neurons exhibiting red fluorescence in the posterior VTA. (B) Left: Bath application of $100 \mathrm{mM}$ EtOH reduced sIPSC frequency in vehicle-treated but not D034-treated slices. Right: Example traces of sIPSC response to $100 \mathrm{mM}$ EtOH in vehicle- or D034-treated slices. (C) Relative proportions of sIPSC frequency (Freq.) response to $100 \mathrm{mM} \mathrm{EtOH} \mathrm{(defined} \mathrm{as} \geq 10 \%$ change). D034 treatment reduced the proportion of cells that exhibited a decrease in sIPSC frequency. One to 3 cells per mouse were included in analyses. Vehicle, $n=9$ mice; D034, $n=10$ mice; 15 mice total (both conditions sampled from a subset of 4 mice). B: Data analyzed by 2-way ANOVA, followed by a Holm-Šidák test for multiple comparisons between baseline and EtOH application. C: Data analyzed by Fisher's exact test for proportion of cells exhibiting reduced sIPSC frequency after EtOH application. Sample size $n$ (cells), $P$, and $F$ values for $\mathrm{EtOH} \times \mathrm{DO} 34$ interaction, and significance for post hoc multiple comparisons are reported in the graphs $\left({ }^{*} P<0.05\right)$.

ed daily with DO34 $(50 \mathrm{mg} / \mathrm{kg})$ for 6 days, starting the day before the first drinking session to maintain consistent DO34 exposure during the intermittent drinking period. DO34 caused a nonsignificant $(P=0.055)$ reduction in $\mathrm{EtOH}$ preference, and $\mathrm{EtOH}$ preference was significantly lower during DO34 treatment when compared with the recovery week after an additional 2BC-vapor exposure cycle (Figure 3, D and E). DO34 treatment significantly reduced EtOH consumption during the treatment week, and consumption recovered to baseline following an additional cycle of 2BC-vapor treatment (Figure 3, F and G). DO34 treatment also caused a small but significant reduction in total fluid consumption during the treatment week; however, fluid intake remained significantly decreased compared with baseline after recovery (Supplemental Figure 6, D and E). These data suggest that DAGL inhibition may be a broadly applicable therapeutic strategy at various stages of AUD, including late-stage dependence, as indicated by the results of the CIE exposure experiments.

Pharmacological DAGL inhibition does not precipitate negative affective behaviors during late chronic EtOH drinking. 2-AG signaling is an important modulator of anxiety- and depression-related behaviors $(7-9,31,32)$, and depletion of 2-AG produces anxiety-like phenotypes and exacerbates the affective consequences of stress exposure $(9,23,32,33)$. Additionally, 2-AG augmentation has been shown to mitigate anxiety-like behaviors in a rodent model of EtOH withdrawal (34). When considering these data in the context of DAGL inhibition as an AUD treatment strategy, a potential problem is that pharmacological depletion of 2-AG may produce affective disturbances similar to those previously observed with the $\mathrm{CB}_{1}$ receptor inverse agonist rimonabant (20). We therefore wanted to examine the potential affective side effect profile of DAGL inhibition by observing the effects of DAGL inhibition on anxiety- and depression-like behaviors in animals after chronic alcohol exposure. To test this, we exposed female mice to 6 weeks of 2BC drinking at 10\% EtOH (or water as a control) and subjected them to a series of behavioral tests after treatment with vehicle or DO34 $(50 \mathrm{mg} / \mathrm{kg}) 2$ hours prior to each behavioral test. These mice were tested in an open-field (OF) test, elevated zero maze (EZM), tail suspension test (TST), light-dark box, and 3-chamber social interaction assay (Supplemental Figure 7A). DO34 treatment decreased the distance traveled in the center as a percentage of total OF distance in water-treated control but not EtOH-treated mice (Supplemental Figure 7B). Additionally, DO34 treatment increased EZM open-arm entries in EtOH mice, but not in water control mice, and total distance in the EZM was increased by DO34 treatment in both water- and EtOH-exposed mice (Supplemental Figure 7C). In the TST, DO34 increased immobility time in water-exposed control mice, but decreased immobility time in EtOH-treated mice, supported by an interaction between EtOH and DO34 (Supplemental Figure 7D). There was no effect of DO34 treatment or EtOH history on anxiety-like behaviors in the light-dark box or in social function as measured by the 3-chamber social interaction test (Supplemental Figure 7, E and F).

We repeated this experiment in an additional cohort of mice drinking EtOH at 20\% for 6 weeks, followed by behavioral testing in novelty-induced feeding suppression (NIFS) test, forced swim test (FST), and elevated plus maze (EPM) assay. There was no effect of DO34 treatment on latency to first bite in the NIFS test, immobility time in the FST, or open-arm entries in the EPM in either water- or EtOH-exposed mice (Figure 3, H-J). DO34 caused a small but significant increase in immobility time in the EPM in EtOH-drinking mice (Figure 3K). These data agree with the 10\% EtOH cohort behavioral data, collectively demonstrating a lack of anxiety- or depression-like phenotypes in chronically drinking mice treated with DO34.

Pharmacological DAGL inhibition reduces reinstatement of EtOH drinking. Another major obstacle to effective AUD treatment is susceptibility to relapse after abstinence (35). We therefore used a model of EtOH reinstatement to test the effects of DO34 treatment on the potential for relapse after $\mathrm{EtOH}$ abstinence. Mice were subjected to our 2BC paradigm for 5 weeks (at 10\% EtOH), followed by a 10-day withdrawal period, during which EtOH bottles were replaced with water. After the withdrawal period, mice were reexposed to $10 \% \mathrm{EtOH}$ for 9 days. Mice were treated with vehicle or DO34 $(50 \mathrm{mg} / \mathrm{kg}$ ) for 7 consecutive days, starting 2 days 
prior to the initiation of EtOH reinstatement. DO34-treated mice showed significantly lower EtOH preference (Figure 4A) and consumption (Figure 4B) compared with vehicle-treated mice upon EtOH reexposure. DO34 treatment concomitantly increased total fluid consumption (Supplemental Figure 8), again demonstrating that the DO34-driven decrease in EtOH consumption was not due to off-target reductions in fluid intake.

Pharmacological DAGL inhibition does not precipitate negative affective behaviors during protracted abstinence from $\mathrm{EtOH}$ drinking. Although DO34 effectively mitigates reinstatement of alcohol drinking after abstinence, affective disturbances are particularly problematic during protracted abstinence from alcohol drinking $(35,36)$. Accordingly, this time point may be of notable concern for potential affective side effects of DAGL inhibition. To examine the effects of DO34 treatment after protracted abstinence from $\mathrm{EtOH}$, we exposed female mice to our 2BC paradigm with $20 \% \mathrm{EtOH}$ (or water as a control) for 6 weeks and subjected them to forced abstinence for 2 weeks (Figure $4, \mathrm{C}-\mathrm{E}$ ). Behavioral tests were matched to those performed on the cohort drinking $20 \% \mathrm{EtOH}$ represented in Figure 3, H-K (NIFS, FST, EPM). Mice were treated with vehicle or DO34 (50 $\mathrm{mg} / \mathrm{kg}$ ) on testing days 2 hours prior to each behavioral test. EtOH-withdrawn animals treated with vehicle exhibited a greater latency to first bite relative to water-drinking control mice in the NIFS assay, suggesting an anxious phenotype produced by withdrawal from EtOH (Figure 4F). DO34 reduced this latency in EtOH-withdrawn mice, with no effect on water-drinking mice, and supported by an interaction between EtOH and DO34 (Figure 4F). DO34 reduced immobility time in the FST in both water- and EtOH-drinking mice (Figure 4G). In the EPM assay, DO34 increased open-arm entries and decreased immobility time only in EtOH-withdrawn mice, again supported by an interaction between EtOH and DO34 (Figure 4, H and I). These data suggest that DO34 does not produce an anxiety- or depression-like phenotype during protracted alcohol withdrawal and, in contrast, may exhibit anxiolytic or antidepressant properties in some contexts. Although there was variability between the withdrawal and late drinking cohorts with regard to the DO34 effects on immobility time in the FST (Figure 3I and Figure 4G) and EPM (Figure 3K and Figure 4I), these may be due to inter-cohort and inter-experimenter variability; and nonetheless, these data sets collectively suggest low risk for adverse affective side effects from DAGL inhibition after a history of EtOH consumption or protracted abstinence in these mouse models.

DO34 blocks EtOH-driven disinhibition of putative dopamine neurons in the posterior ventral tegmental area. The mitigating effect of DO34 on alcohol consumption suggests a role for 2-AG mobilization in driving alcohol seeking. EtOH stimulates the activity of ventral tegmental area (VTA) dopamine neurons in a manner that requires eCB signaling (16). It has been previously reported that inhibition of $\mathrm{CB}_{1}$ receptors in the posterior (but not anterior) VTA reduces alcohol intake (37). In line with these findings, EtOH reduces inhibitory GABA transmission onto putative dopamine neurons in the posterior VTA (38), and VTA GABA transmission is regulated by eCB signaling (39-42). Furthermore, it has been reported that DAGL lipase signaling disinhibits dopamine neurons by suppressing GABA release following chronic nicotine exposure
(39), suggesting that EtOH may stimulate a similar, eCB-mediated disinhibitory mechanism in these cells. To test whether DAGL regulates alcohol-induced inhibition of VTA GABAergic transmission, we used tyrosine hydroxylase:Ai14 (TH:Ai14) mice expressing Td-tomato in $\mathrm{TH}^{+}$cells to conduct fluorescence-assisted electrophysiological recordings of spontaneous inhibitory postsynaptic currents (sIPSCs) from putative dopamine $\left(\mathrm{TH}^{+}\right)$neurons in posterior midbrain slices (Figure $5 \mathrm{~A}$ ). Acute bath application of EtOH $(100 \mathrm{mM})$ decreased the frequency of sIPSCs onto the majority of cells recorded, as previously reported (ref. 38 and Figure $5 \mathrm{~B}$ ). Incubation of slices in $2.5 \mu \mathrm{M}$ DO34 abolished this effect (Figure 5B) and significantly decreased the proportion of cells that exhibited a reduction in sIPSC frequency in response to EtOH application (Figure 5C). Additionally, EtOH induced a small but significant reduction in sIPSC amplitude in DO34-treated slices (Supplemental Figure 9).

Although these data suggest that EtOH mobilizes 2-AG at posterior VTA GABA synapses, there is no direct evidence of EtOH-stimulated increases in VTA 2-AG levels, as has been shown in the nucleus accumbens $(10,11)$. To attempt to address this, we injected mice with an i.p. bolus of EtOH $(3 \mathrm{~g} / \mathrm{kg})$ and 30 minutes later collected acute midbrain tissue punches containing the VTA. Mass spectrometry analysis of midbrain punches showed no effect on midbrain 2-AG levels (Supplemental Figure 10A). We repeated this experiment in a separate cohort of mice after 6 weeks of voluntary drinking in the $2 \mathrm{BC}$ drinking paradigm at $10 \% \mathrm{EtOH}$. These punches were taken at the initiation of the dark cycle to attain maximal alcohol intake based on 2BC drinking time course measurements previously obtained from another cohort (Supplemental Figure 10B). This method again did not show any effect on midbrain 2-AG levels (Supplemental Figure 10C). This could be due to technical limitations and the lack of anatomical specificity afforded by bulk punches from mouse brain tissue, as our punches undoubtedly contained both anterior and posterior VTA (for the specificity of posterior VTA in the effects of $\mathrm{CB}_{1}$ on alcohol intake, see ref. 37), as well as portions of other adjacent midbrain structures. This question could be better addressed in future studies with an alternative approach that offers greater spatial resolution, such as use of cannabinoid-sensitive biosensors. Despite these technical limitations, our electrophysiological data functionally suggest that DO34 could decrease positive reinforcement-driven voluntary $\mathrm{EtOH}$ consumption via prevention of $\mathrm{EtOH}$-induced reductions in VTA GABA transmission and subsequent increases in VTA dopamine neuron activity.

\section{Discussion}

Here we show that genetic and pharmacological inhibition of DAGL activity reduced alcohol consumption and preference across a range of distinct drinking models. Specifically, pharmacological DAGL inhibition and DAGL $\alpha$ genetic knockout in mice led to reduced voluntary alcohol consumption and preference under a continuous access model. Importantly, pharmacological DAGL inhibition also reduced alcohol consumption and preference in an aversion-resistant drinking model; in a dependence-like model in which chronic intermittent vapor exposure is leveraged to drive an escalation in voluntary alcohol consumption; and in a model of reinstatement drinking. In line with our data, DAGL inhibition by 
DO34 has been previously shown to reduce operant responding for alcohol (43). The magnitude of effect on EtOH intake observed after DAGL inhibition was comparable to or greater than those observed with clinically available treatments such as naltrexone $(44-46)$ and acamprosate $(46,47)$ in similar rodent models. Moreover, pharmacological DAGL inhibition did not affect sucrose preference, suggesting that the effect was not generalized to natural reward, although female DAGL $\alpha$-KO mice do exhibit reductions in sucrose preference under some conditions (23). Although DO34 inhibits both the $\alpha$ and $\beta$ isoforms of DAGL (22), the ability of DAGL $\alpha$ genetic knockout to reduce alcohol consumption suggests that the $\alpha$ isoform may be the relevant molecular target contributing to the efficacy of DO34. Furthermore, we found that a control compound, DO53 - which does not inhibit DAGL $\alpha$ or $-\beta$ but does inhibit a number of off-targets also inhibited by DO34 (22) - produced a transient suppression of alcohol intake that was paralleled by a reduction in nonspecific fluid intake and no effect on alcohol preference. This effect may be due to activity at shared off-targets or unique off-targets of DO53, such as ABHD3/6 (22). Regardless of the precise mechanisms by which DO53 transiently suppresses nonspecific fluid intake, it did not affect alcohol preference or alcohol consumption after repeated injection, providing an additional degree of confidence that the effects of DO34 on alcohol consumption and preference were mediated via DAGL inhibition. Overall, these data suggest that targeting DAGL $\alpha$ may represent an effective approach to the treatment of AUD across a spectrum of severity, and development of potent and selective DAGL $\alpha$ inhibitors should remain a high priority. The DAGLA transcript is known to be preferentially expressed within the human brain, while $D A G L B$ is more widely expressed (see Supplemental Figure 11). Accordingly, DAGL $\alpha^{-/-}$mice have been shown to have reduced brain 2-AG levels, with no difference in peripheral 2-AG relative to WT littermates (23), collectively suggesting that selective targeting of DAGL $\alpha$ may avoid adverse somatic effects; however, this remains to be determined experimentally.

Another key finding of the present work is that DO34 did not increase anxiety- or depression-like behaviors in mice exposed to chronic alcohol or during protracted abstinence. In chronically drinking mice, DO34 reduced immobility in the TST, while it increased immobility in water-drinking controls. These data exemplify the notion that the effects of DO34 are dependent on alcohol history and could explain, in part, the apparently contradictory results relative to previously published work that would predict an increase in affective behaviors $(9,33)$. For example, DO34 increases anxiety-like behaviors in mice exposed to acute stress (33) and impairs extinction of conditioned fear memories (48). Moreover, DAGL $\alpha$-KO mice exhibit anxiety-like behaviors under some, but not all, conditions, and some reports are conflicting. For example, a robust anxiety-like phenotype was found in DAGL $\alpha-\mathrm{KO}$ mice in one study using a light-dark box assay (23), but not in a later study using a similar assay (49). Furthermore, DO34 exerted mild anxiolytic effects during protracted abstinence, which was unexpected given the anxiogenic effects of DO34 observed after stress exposure (33). These data again highlight the notion that the function of 2-AG signaling in modulating anxiety- and depression-like phenotypes is highly dependent on context, such as alcohol use history. Interest- ingly, we recently showed that augmentation of 2-AG levels via inhibition of the 2-AG-degrading enzyme monoacylglycerol lipase (MAGL) also had anxiolytic effects $(9,33)$, and MAGL inhibition has exhibited anxiolytic effects in alcohol withdrawal as well (34). This apparently paradoxical finding has also been observed in other models; for example, both inhibition (48) and augmentation (50) of 2-AG signaling can facilitate acute fear responses in conditioned fear paradigms. These data collectively suggest that bidirectional manipulations of 2-AG levels can have overlapping effects in some cases, and the behavioral effects of pharmacological 2-AG modulation may be highly context dependent. Furthermore, there are several genetic variants that strongly alter DAGL $\alpha$ expression in the brain (see Supplemental Figure 12 for one example, rs11604261), demonstrating that the baseline expression of DAGL $\alpha$ can vary across individuals. This suggests that variation within the DAGLA gene may demonstrate correlations with susceptibility to AUD or other neuropsychiatric disorders or serve as a biomarker for predicting responsiveness to pharmacological DAGL inhibition. Largescale genetic studies are needed to test this hypothesis.

eCB signaling has been shown to regulate VTA dopamine neuron activity via suppression of afferent GABAergic drive (39-42). For example, 2-AG-mediated suppression of GABA transmission onto VTA DA neurons is weaker in Sardinian alcohol-preferring rats relative to nonpreferring rats $(40,42)$, an effect mediated by enhanced 2-AG degradation (40). These data suggest that 2-AG can promote alcohol preference via activity-dependent suppression of GABAergic transmission onto VTA dopamine neurons. Based on these data, we tested the hypothesis that pharmacological DAGL inhibition could affect alcohol modulation of GABAergic transmission onto VTA DA neurons, the firing rate of which has been shown to increase in response to exogenous alcohol (16). Consistent with these data, ex vivo alcohol application to VTA brain slices decreased IPSC frequency into posterior VTA dopamine neurons, as shown previously (38). This effect was blocked by DO34, suggesting that DAGL inhibition could reduce alcohol consumption and preference by reducing alcohol-stimulated dopamine neuron activity via maintaining high relative levels of GABAergic inhibition. Similar mechanisms have been proposed to underlie the effects of DAGL signaling on nicotine activation of VTA dopamine neurons (39). While these data suggest that DAGL inhibition could reduce positive reinforcement-driven alcohol consumption by reducing alcohol-induced activation of VTA dopamine neurons, negative reinforcement-driven mechanisms are thought to mediate alcohol seeking and drinking under dependent conditions (28). Although DO34 was able to reduce alcohol consumption in a dependent-like drinking model, the underlying synaptic mechanisms subserving these effects may indeed be different. Future studies should be aimed at elucidating the distinct mechanisms by which DAGL inhibition affects positive versus negative reinforcement-driven alcohol drinking.

Alterations in other molecular targets that interact with DAGL have been implicated in the pathophysiology of AUD as well, most notably metabotropic glutamate receptor 5 (mGlu5), which couples to DAGL function through the canonical $\mathrm{G}_{\mathrm{q}} /$ phospholipase $\mathrm{C} \beta / \mathrm{DAGL}$ pathway. mGlu5 has also been posited as 
a potential therapeutic target for AUD, as several studies have shown that inhibition of mGlu5 has effects similar to those of DAGL inhibition with regard to both alcohol consumption (51, 52) and negative affect (refs. 53, 54; see ref. 55 for review), and mGlu5 expression levels are altered by chronic drinking and abstinence $(54,56)$. Chronic alcohol exposure has also been shown to disrupt group I mGlu receptor-mediated depression of glutamate release in the bed nucleus of the stria terminalis (57), the maximal magnitude of which has been shown to be $\mathrm{CB}_{1}$ receptor dependent (58), although the specific role of 2-AG in this effect has not been experimentally demonstrated. The relationship between altered mGlu5 and DAGL function after chronic alcohol exposure and the mechanisms by which DO34 exerts unexpected anxiolytic effects remain to be determined.

In summary, our data present a key role for DAGL signaling in voluntary alcohol drinking and posit targeting 2-AG synthesis as a promising pharmacotherapeutic mechanism for the treatment of AUD. The finding that DO34 does not produce affective phenotypes (and exhibits mild anxiolytic effects in some cases) further highlights the therapeutic potential of DAGL as a target for AUD and is especially pertinent given the neuropsychiatric side effects of $\mathrm{CB}_{1}$ receptor blockade (20). The various models in which we tested the effects of DO34 address several key hallmarks of AUD that serve as barriers to current treatments, including aversion resistance, susceptibility to relapse, and dependence-like drinking. The ability of DO34 to reduce drinking in all these contexts suggests that DAGL inhibition may have therapeutic utility for treating the broad spectrum of early- to late-stage, severe AUD, which could have broad implications. Future studies should be aimed at determining the cellular and synaptic mechanisms underlying the effects of DO34 on early- versus late-stage, dependence-like drinking, as well as on the development of more selective DAGL $\alpha$ inhibitors to further test this hypothesis and ultimately lead to the discovery of safer and more effective therapies for AUD.

\section{Methods}

\section{Subjects}

C57BL/6J male and female mice between 6 and 8 weeks of age at the beginning of experiments were used. DAGL $\alpha^{-/-}$male and female mice were generated by disruption of exon 8 and were maintained on a C57BL6/N background by interbreeding with DAGL $\alpha^{+/-}$mice. Further details are provided in ref. 23. TH reporter (TH:Ai14) mice were generated by crossing TH-Cre mice (The Jackson Laboratory, 008601) with Ai14 mice (The Jackson Laboratory, 007914) for Cre-dependent Td-tomato expression in $\mathrm{TH}^{+}$cells to label catecholaminergic neurons. All mice were group housed on a 12-hour light/12 -hour dark cycle (lights on at 6:00 am) with food and water available ad libitum. All behavioral testing was performed between 6:00 am and 6:00 pm.

Drugs and treatment. The DAGL inhibitor DO34 (50 mg/kg; Glixx Laboratories Inc.), control compound DO53 (50 mg/kg; synthesized in-house; see Supplemental Figure 1), MAGL inhibitor JZL184 (10 $\mathrm{mg} / \mathrm{kg}$; Cayman Chemical), pyrazole (68 mg/kg; Sigma-Aldrich), and quinine hydrochloride (0.01, 0.03, and $0.1 \mathrm{~g} / \mathrm{L}$; Millipore) were used. DO34 and DO53 (or vehicle control) were administered by i.p. injection at a volume of $10 \mathrm{~mL} / \mathrm{kg}$ in a formulation containing EtOH:kolliphor:saline (1:1:18; Pharmco, Sigma-Aldrich, Hospira). JZL184 (or vehicle control) was administered by i.p. injection at a volume of 1 $\mathrm{mL} / \mathrm{kg}$ in DMSO (Sigma-Aldrich D8414). EtOH + pyrazole solutions for vapor chamber experiments were prepared in saline and injected i.p. at a dose of $1.6 \mathrm{~g} / \mathrm{kg} \mathrm{EtOH}+68 \mathrm{mg} / \mathrm{kg}$ pyrazole. Drug pretreatment times were 2 hours prior to behavioral testing or 30 minutes prior to vapor chamber sessions. Drugs for EtOH drinking experiments were injected 2 hours before the start of the dark cycle. Quinine hydrochloride (Millipore) was added to the drinking water or EtOH bottles at varying concentrations. 190-proof American Chemical Society (ACS)/United States Pharmacopeia (USP) grade grain-derived EtOH was used to make EtOH solutions.

\section{BC EtOH drinking paradigm}

Mice were singly housed and acclimatized for 5-7 days in 2BC cages. Mice had access to 2 sippers throughout the experiment. For EtOH-drinking mice, EtOH concentration was slowly increased from $3 \%$ to $10 \%$ or $20 \%$ as shown in Figure $1 \mathrm{~A}$ and maintained on the respective solutions for the duration of the experiment. Water and $\mathrm{EtOH}$ intake were monitored daily, after 4 days, or weekly, depending on the experimental timeline. For the intermittent-access model (see Figure 2E), mice were given intermittent access to alcohol (10\% on week $1,15 \%$ thereafter) 3 days per week for 24 -hour periods on alternating days. Whenever mice were given pharmacological treatments, water intake, EtOH intake, and body weights were measured daily. Food was provided ad libitum throughout the alcohol-drinking paradigm. EtOH-naive mice (control) were housed in the same conditions, but 2 bottles of water remained in their cage until the day of experiment. EtOH preference was determined as ([EtOH intake $(\mathrm{g})] /[$ total fluid intake $(\mathrm{g})]) \times 100)$. EtOH consumption was determined as ([EtOH intake $(\mathrm{g}) /$ mice body weight $(\mathrm{kg}) /$ number of days] $\times$ EtOH concentration $\times$ EtOH density $(0.816 \mathrm{~g} /$ $\mathrm{mL})$ ). Drip control cages were used to calculate water and EtOH drip and subtracted from intake values.

\section{Aversion-resistant EtOH drinking model}

After 4 weeks of EtOH drinking in the $2 \mathrm{BC} \mathrm{EtOH}$-drinking paradigm, quinine was added to either water (control mice) or EtOH bottles (EtOH group) at various concentrations (0.01, 0.03, and $0.1 \mathrm{~g} / \mathrm{L}$ ). $\mathrm{EtOH}$ quinine and water quinine preference and consumption were calculated as described above.

\section{EtOH vapor inhalation treatment for $\mathrm{CIE}$ exposure}

For CIE exposure, female C57BL6/J mice were exposed to vapor inhalation for 16 hours per day, 4 days per week, on alternating weeks as shown in Figure 4C. Mice were given i.p. injections of EtOH $(1.6 \mathrm{~g} / \mathrm{kg})$ + pyrazole (alcohol dehydrogenase inhibitor, $68 \mathrm{mg} / \mathrm{kg}$ ) 30 minutes prior to the start of each session. Mice were then placed in a chamber with volatilized EtOH (18-22 mg/L) for 16 hours. Mice had ad libitum access to food and water for the duration of the session. These procedures were similar to those done previously (59), which were able to produce blood EtOH levels of 150-185 mg/dL. Following 4 days of vapor treatment, mice went through 72 hours of abstinence before returning to the intermittent $2 \mathrm{BC}$ paradigm described above.

\section{EtOH reinstatement drinking}

After 5 weeks of EtOH drinking in the 2BC EtOH-drinking paradigm, $\mathrm{EtOH}$ bottles were replaced with water bottles for 10 days. After 10 
days, EtOH was provided to study reinstatement of EtOH drinking behavior. EtOH preference and consumption were calculated as described above.

\section{Behavioral experiments}

NIFS. The NIFS assay was performed as described previously (31). Briefly, mice were deprived of food for a 48-hour period, with access to food from hours 23 to 25 . Following food deprivation, mice were acclimated to the testing room for at least 2 hours prior to placement in a brightly lit (250-300 lux) $50 \times 50 \mathrm{~cm}$ arena with a single food pellet in the center of the arena. Mouse movement and behavior were tracked with an overhead camera using ANY-maze software (Stoelting). Mice remained in the arena until taking their first bite of the food pellet. Mice were given ad libitum access to food after testing.

Marble burying test. For the marble burying test, empty cages without food or water were filled with $5 \mathrm{~cm}$ fresh Diamond Fresh Soft Bedding (Envigo) and 20 marbles (5 rows, 4 columns) placed on top of the bedding. Mice were placed in the cage for 30 minutes, then removed. Marbles were considered buried if two-thirds of the marble was covered by bedding. Marbles were counted manually by an experimenter who was blinded to the treatment condition. Light intensity in the room was 200-250 lux.

FST. FST was performed as described previously (31). Briefly, mice acclimated to the testing room for at least 2 hours were placed in a cylinder containing water $\left(23^{\circ} \mathrm{C}-25^{\circ} \mathrm{C}\right)$ at a level such that mice could not touch the bottom or the top. The test lasted for 6 minutes and filmed via overhead camera, and was scored by an observer blinded to treatment. Immobility time was analyzed during the last $4 \mathrm{~min}$ utes of the test.

EPM. The EPM assay was performed in a manner similar to that described previously (32). The apparatus consisted of 2 open arms $(30 \times 10 \mathrm{~cm})$ and 2 closed arms $(30 \times 10 \times 20 \mathrm{~cm})$ that met at a center junction $(5 \times 5 \mathrm{~cm})$ and was elevated $50 \mathrm{~cm}$ above the floor. Light intensity in the open arms was 200-250 lux and less than 100 lux in the closed arms. Mice were placed in the center of the apparatus facing an open arm and allowed to freely explore for 6 minutes. Movement and behavior were tracked via an overhead camera and ANY-maze software.

OF. For OF testing, exploration of a novel OF arena contained within a sound-attenuating chamber was monitored for 30 minutes $(27.9 \times 27.9 \times 20.3 \mathrm{~cm}$; MED Associates Inc. MED-OFA-510 $)$. The walls of the open-field arena were made of clear plexiglass; this arena was contained within an opaque, sound-attenuating chamber. Beam breaks from 16 infrared beams were recorded by an Activity Monitor v5.10 (MED Associates Inc.) to monitor position and behavior.

3-Chamber social interaction. Social behavior was tested in a 3-chamber acrylic arena with opaque outer walls and clear walls separating each $20 \times 40 \mathrm{~cm}$ chamber, which contained $10 \mathrm{~cm}$ sliding doors for introduction of the test mouse into the middle chamber. Testing was performed under low lighting conditions ( 40 lux), and mice were acclimated to this lighting for at least 1 hour before testing began. The doors were removed, and the test mouse was allowed to explore the apparatus, which contained 1 empty inverted wire pencil cup in each outer chamber, for 10 minutes. The mouse was then coaxed back into the middle chamber, and the doors were replaced. The target mouse (a female WT C57BL/6J mouse that was age matched and previously acclimated to the pencil cup for 30 minutes) was then placed under one of the pencil cups, and the doors were removed, allowing the test mouse to explore the entire apparatus for 5 minutes. The mouse was monitored by an overhead camera and ANY-Maze software (Stoelting), and the time spent in each chamber was measured. Preference for the social target was determined within each group by comparing the time spent in the target-mouse chamber to the time spent in the empty pencil cup chamber using a paired 2-tailed Student's $t$ test. Mice that did not spend significantly more time in the target-mouse chamber were considered deficient in sociability.

EZM. The EZM (San Diego Instruments) is a white annular platform divided into 4 equal quadrants. It consisted of 2 open arms and 2 closed arms. The outer and inner diameters of the EZM were 60.9 $\mathrm{cm}$ and $50.8 \mathrm{~cm}$, respectively. The apparatus was elevated $60.9 \mathrm{~cm}$ from the floor. Light levels in the open arms were approximately 200 lux, while those in the closed arms were less than 100 lux. Mice were placed in the closed arm of the maze and allowed to explore for $5 \mathrm{~min}$ utes. ANY-maze video tracking software was used to monitor and analyze behavior during the test.

TST. In the TST test, each mouse was suspended from its tail by use of adhesive tape on a flat metal plate for 6 minutes. In order to prevent animals observing or interacting with each other, each mouse was suspended within a 4 -walled rectangular compartment $(33 \times 31.75 \times$ $33 \mathrm{~cm}$ ). The mouse was suspended in the middle of the compartment, and the width and depth were sufficiently sized so that the mouse could not make contact with the walls. Immobility time was automatically measured by Med Associates Inc. tail suspension software.

Light-dark box test. The light-dark test was performed as previously described (9). Mice were individually placed in sound-attenuating chambers $(27.9 \times 27.9 \mathrm{~cm}$; MED Associates Inc. MED-OFA-510) containing dark box inserts that split the chamber into light (250-400 lux) and dark ( $<5$ lux) halves (Med Associates Inc. ENV-511). Beam breaks from 16 infrared beams were recorded by Activity Monitor v5.10 (Med Associates Inc.) to monitor position and behavior during the 10-minute testing period.

\section{Brain tissue preparation and mass spectrometry}

For acute brain tissue sample collection, mice were sacrificed by cervical dislocation and decapitation, followed by rapid removal of the brain. For half-brain samples, mice were treated with DO34 (50 mg/ $\mathrm{kg}$ ) for 4 days. Two hours after the final treatment, whole brains were removed and snap frozen in 2-methylbutane (Sigma-Aldrich) on dry ice, and quickly hemisected before being stored at $-80^{\circ} \mathrm{C}$ for further processing. Of note, brains collected for the analysis shown in Figure 2 were from the mice that underwent behavioral testing represented in Figure 3, H-K. For midbrain tissue punches, brains were blocked around the VTA (1 mm thick section) and snap frozen by placing the blade and tissue on an aluminum block in dry ice. Midbrain punches $(1.5-2.0 \mathrm{~mm})$ were then taken from the center of the section and stored at $-80^{\circ} \mathrm{C}$ for further processing.

Liquid chromatography and mass spectrometry performed in a manner similar to that previously reported (60). Briefly, samples were sonicated in $300 \mu \mathrm{L}$ homogenization solution and placed in a bath sonicator for up to 2 minutes and incubated at $-20^{\circ} \mathrm{C}$ overnight. The following day, samples were centrifuged at $2060 \mathrm{~g}$ for 12 minutes at $4^{\circ} \mathrm{C}$. Supernatant was collected and dried under nitrogen. Afterward, samples were reconstituted by the addition of $60 \mu \mathrm{L}$ methanol fol- 
lowed by $30 \mu \mathrm{L}$ distilled water and vortexed. Finally, samples were centrifuged at $2060 \mathrm{~g}$ for 12 minutes at $4^{\circ} \mathrm{C}$ if they were cloudy and/or had visible particulate matter. Liquid chromatography-tandem mass spectrometry (LC-MS/MS) analysis was performed on a Shimadzu Nexera X2 system in-line with SCIEX QTRAP 6500. Samples were analyzed using the Analyst software program and then analyzed for significance with GraphPad Prism. Data were normalized to tissue mass and presented as either $\mathrm{pmol} / \mathrm{g}$ tissue or $\mathrm{nmol} / \mathrm{g}$ tissue. Detection methods for analytes and internal standards are presented in Supplemental Table 1.

\section{Blood EtOH concentration measurements}

Female WT mice were injected with DO34 $(50 \mathrm{mg} / \mathrm{kg})$ or vehicle and 2 hours later given an i.p. bolus of EtOH $(3 \mathrm{~g} / \mathrm{kg})$. Thirty minutes after EtOH injection, mice were anesthetized under isoflurane and decapitated for trunk blood collection. Samples were allowed to coagulate at room temperature, then placed on ice until undergoing centrifugation at $2100 \mathrm{~g}$ for 15 minutes at $4^{\circ} \mathrm{C}$. Serum supernatant samples were extracted and used for evaluation of blood EtOH concentration per the protocol provided in the kit (Pointe Scientific A7504-39). The standard EtOH concentrations were obtained from a calibration kit (Cerilliant E-034), and the assay was run in a 96-well plate and read on a GloMax-Discover plate reader (Promega GM3000).

\section{Electrophysiology experiments}

Male and female TH:Ai14 mice (described above) were used for electrophysiological experiments. Acute slice preparation was performed as described previously (33). Coronal midbrain slices were cut at 200 $\mu \mathrm{m}$. Following recovery, slices were transferred to a recording chamber and perfused at a rate of $2-3 \mathrm{~mL} / \mathrm{min}$ with oxygenated artificial cerebrospinal fluid (ACSF; $31^{\circ} \mathrm{C}-33^{\circ} \mathrm{C}$ ) recording solution consisting of (in mM): $113 \mathrm{NaCl}, 2.5 \mathrm{KCl}, 1.2 \mathrm{MgSO}_{4} \cdot 7 \mathrm{H}_{2} \mathrm{O}, 2.5 \mathrm{CaCl}_{2} \cdot 6 \mathrm{H}_{2} \mathrm{O}, 1$ $\mathrm{NaH}_{2} \mathrm{PO}_{4}, 26 \mathrm{NaHCO}_{3}, 20$ glucose, 3 Na-pyruvate, and $1 \mathrm{Na}$-ascorbate. $\mathrm{TH}^{+}$cells were visually identified by their red fluorescence. Whole-cell recordings of sIPSCs were performed in voltage clamp configuration at $-70 \mathrm{mV}$ in the presence of $10 \mu \mathrm{M} \mathrm{CNQX}$ and 30 $\mu \mathrm{M}$ D-AP5. Borosilicate glass pipettes (3-6 M $\Omega$ ) were filled with the intracellular pipette solution consisting of (in $\mathrm{mM}$ ): $125 \mathrm{KCl}, 4 \mathrm{NaCl}$, 10 HEPES, 4 MgATP, 0.3 NaGTP, 10 Na-phosphocreatine, and 0.2 EGTA. Following break-in, all cells were allowed to dialyze with the pipette solution for 3 minutes prior to recordings. Cells with an access resistance of $\leq 25 \mathrm{M} \Omega$ were included in analyses. DO34 was dissolved in DMSO stocks at $10 \mathrm{mM}$ and diluted in ACSF for a final concentration of $2.5 \mu \mathrm{M}$. DO34-treated slices were incubated for a minimum of 45 minutes prior to recordings. sIPSC baselines were recorded for 2 minutes, followed by 10-minute bath application of $100 \mathrm{mM} \mathrm{EtOH}$. Cells were recorded for an additional 2 minutes in the continued presence of EtOH. Recordings were performed using a MultiClamp 700B amplifier and Clampex software (Molecular Devices). Traces were analyzed using pCLAMP 10 software (Molecular Devices).

\section{Statistics}

Drinking time course experiments examining 2 groups (genotype or drug vs. vehicle) were analyzed via a repeated-measure 2-way ANOVA followed by a Holm-Šidák multiple-comparison test between groups at all time points. Single-group drinking time course experiments were analyzed via a 1-way ANOVA for time points noted in the figure legends, followed by a Holm-Šidák multiple-comparison test referenced to baseline before drug treatment. Behavioral data were analyzed by 2-tailed $t$ test or 2-way ANOVA followed by a Holm-Šidák multiple-comparison test between all groups as noted in the figure legends. Electrophysiology data were analyzed by 2-way ANOVA followed by a Holm-Šidák multiple-comparison test between baseline and EtOH application or by Fisher's exact test. All statistical analyses were conducted using GraphPad Prism 7 (GraphPad Software). For behavioral studies, $n$ values represent biological replicates, defined as data derived from a single mouse. Summary data are presented as mean \pm SEM unless otherwise noted. Significance was defined as $P<0.05$ throughout. $F$ and $P$ values for ANOVA analyses are indicated within figure panels, while post hoc significance levels are indicated above individual bars or time points. Testing was counterbalanced, but no randomization was performed, and sample sizes were derived empirically during the course of the experiments and guided by our previous work using these assays.

\section{Study approval}

All studies were carried out in accordance with the Guide for the Care and Use of Laboratory Animals (National Academies Press; 2011) and approved by the Vanderbilt University Institutional Animal Care and Use Committee (M1600213-01, M1800046, M1900114-00).

\section{Author contributions}

GB, NDW, and SP conceived the study, designed experiments, and wrote the manuscript. SWC and DGW assisted in the development and optimization of the alcohol-drinking model. GB, NDW, TAP, AA, MA, AJM, KDJ, SM, and VRM performed experiments and acquired and analyzed data in the laboratories of SP, DJW, and CAS. MJU synthesized the control compound DO53, and PJK and MA ran the mass spectrometry experiments in the laboratory of LJM. DCS acquired gene expression data.

\section{Acknowledgments}

These studies were supported by NIH grants AA026186 (to SP), AA019455 (to DGW), and R00 DA04510 (to CAS); an Alkermes Pathways Research Award, the Brain Research Foundation, and the Whitehall Foundation (to CAS); and Brain and Behavior Research Foundation NARSAD Young Investigator Award 27172 (to SWC).

Address correspondence to: Sachin Patel, Department of Psychiatry and Behavioral Sciences, Medical Research Building IV, Rm 8425B, Vanderbilt University Medical Center, Nashville, Tennessee 37232, USA. Phone: 615.936.7768; Email: sachin.patel@vanderbilt.edu.
1. Grant BF, et al. Epidemiology of DSM-5 alcohol use disorder. JAMA Psychiatry. 2015;72(8):757-766.

2. Kranzler HR, Soyka M. Diagnosis and pharmacotherapy of alcohol use disorder: a review. JAMA. 2018;320(8):815-824.

3. Centers for Disease Control and Prevention.
Excessive Alcohol Use: A Drain on the American Economy. https://www.cdc.gov/alcohol/onlinemedia/infographics/excessive-alcohol-economy. html. Updated December 30, 2019. Accessed November 1, 2020.

4. Evoy KE, et al. National outpatient medication utilization for opioid and alcohol use disorders from 2014 to 2016. J Subst Abuse Treat. 2020;119:108141.

5. Kunos G. Interactions between alcohol and the endocannabinoid system. Alcohol Clin Exp Res. 2020;44(4):790-805 
6. Henderson-Redmond AN, et al. Roles for the endocannabinoid system in ethanol-motivated behavior. Prog Neuropsychopharmacol Biol Psychiatry. 2016;65:330-339.

7. Bedse G, et al. Endocannabinoid signaling in the central amygdala and bed nucleus of the stria terminalis: implications for the pathophysiology and treatment of alcohol use disorder. Alcohol Clin Exp Res. 2019;43(10):2014-2027.

8. Parsons LH, Hurd YL. Endocannabinoid signalling in reward and addiction. Nat Rev Neurosci. 2015;16(10):579-594.

9. Bedse G, et al. Functional redundancy between canonical endocannabinoid signaling systems in the modulation of anxiety. Biol Psychiatry. 2017;82(7):488-499.

10. Caillé S, et al. Specific alterations of extracellular endocannabinoid levels in the nucleus accumbens by ethanol, heroin, and cocaine self-administration. J Neurosci. 2007;27(14):3695-3702.

11. Alvarez-Jaimes L, et al. Chronic ethanol treatment potentiates ethanol-induced increases in interstitial nucleus accumbens endocannabinoid levels in rats. J Neurochem. 2009;111(1):37-48.

12. Basavarajappa BS, et al. Stimulation of cannabinoid receptor agonist 2-arachidonylglycerol by chronic ethanol and its modulation by specific neuromodulators in cerebellar granule neurons. Biochim Biophys Acta. 2000;1535(1):78-86.

13. Basavarajappa BS, et al. Acute ethanol suppresses glutamatergic neurotransmission through endocannabinoids in hippocampal neurons. J Neurochem. 2008;107(4):1001-1013.

14. Vinod KY, et al. Genetic and pharmacological manipulations of the $\mathrm{CB} 1$ receptor alter ethanol preference and dependence in ethanol preferring and nonpreferring mice. Synapse. 2008;62(8):574-581.

15. Marinho EAV, et al. Effects of rimonabant on the development of single dose-induced behavioral sensitization to ethanol, morphine and cocaine in mice. Prog Neuropsychopharmacol Biol Psychiatry. 2015;58:22-31.

16. Perra S, et al. Involvement of the endogenous cannabinoid system in the effects of alcohol in the mesolimbic reward circuit: electrophysiological evidence in vivo. Psychopharmacology (Berl). 2005;183(3):368-377.

17. Soyka M, et al. Cannabinoid receptor 1 blocker rimonabant (SR 141716) for treatment of alcohol dependence: results from a placebo-controlled, double-blind trial. J Clin Psychopharmacol. 2008;28(3):317-324.

18. Pataky Z, et al. Efficacy of rimonabant in obese patients with binge eating disorder. Exp Clin Endocrinol Diabetes. 2013;121(1):20-26.

19. Robinson JD, et al. Pooled analysis of three randomized, double-blind, placebo controlled trials with rimonabant for smoking cessation. Addict Biol. 2018;23(1):291-303.

20. Sam AH, et al. Rimonabant: from RIO to Ban. JObes. 2011;2011:432607.

21. Bisogno $\mathrm{T}$ et al. Cloning of the first sn1-DAG lipases points to the spatial and temporal regulation of endocannabinoid signaling in the brain. JCell Biol. 2003;163(3):463-468.

22. Ogasawara D, et al. Rapid and profound rewiring of brain lipid signaling networks by acute diacylglycerol lipase inhibition. Proc Natl Acad Sci US A. 2016;113(1):26-33.

23. Shonesy BC, et al. Genetic disruption of 2-arachidonoylglycerol synthesis reveals a key role for endocannabinoid signaling in anxiety modulation. Cell Rep. 2014;9(5):1644-1653.

24. Hermanson DJ, et al. Substrate-selective COX-2 inhibition as a novel strategy for therapeutic endocannabinoid augmentation. Trends Pharmacol Sci. 2014;35(7):358-367.

25. Lallemand F, De Witte P. Ethanol induces higher BEC in CB1 cannabinoid receptor knockout mice while decreasing ethanol preference. Alcohol Alcohol. 2005;40(1):54-62.

26. Pruett $S$, et al. Dosage scaling of alcohol in binge exposure models in mice: an empirical assessment of the relationship between dose, alcohol exposure, and peak blood concentrations in humans and mice. Alcohol. 2020;89(89):9-17.

27. Hopf FW, Lesscher HMB. Rodent models for compulsive alcohol intake. Alcohol. 2014;48(3):253-264.

28. Cho S Bin, et al. Positive and negative reinforcement are differentially associated with alcohol consumption as a function of alcohol dependence. Psychol Addict Behav. 2019;33(1):58-68.

29. George O, et al. Recruitment of medial prefrontal cortex neurons during alcohol withdrawa predicts cognitive impairment and excessive alcohol drinking. Proc Natl Acad Sci U S A. 2012;109(44):18156-18161.

30. Becker HC. Animal models of excessive alcohol consumption in rodents. Curr Top Behav Neurosci. 2012;13:355-377.

31. Centanni SW, et al. Endocannabinoid control of the insular-bed nucleus of the stria terminalis circuit regulates negative affective behavior associated with alcohol abstinence. Neuropsychopharmacology. 2019;44(3):526-537.

32. Bluett RJ, et al. Endocannabinoid signalling modulates susceptibility to traumatic stress exposure. Nat Commun. 2017;8:14782.

33. Marcus DJ, et al. Endocannabinoid signaling collapse mediates stress-induced amygdalo-cortica strengthening. Neuron. 2020;105(6):1062-1076.

34. Holleran KM, et al. Ketamine and MAG lipase inhibitor-dependent reversal of evolving depressive-like behavior during forced abstinence from alcohol drinking. Neuropsychopharmacology. 2016;41(8):2062-2071.

35. Driessen M, et al. The course of anxiety, depression and drinking behaviours after completed detoxification in alcoholics with and without comorbid anxiety and depressive disorders. Alcohol Alcohol. 2001;36(3):249-255.

36. Centanni SW, et al. Driving the downward spiral: alcohol-induced dysregulation of extended amygdala circuits and negative affect. Alcohol Clin Exp Res. 2019;43(10):2000-2013.

37. Alvarez-Jaimes L, et al. Regional influence of cannabinoid $\mathrm{CB}_{1}$ receptors in the regulation of ethanol self-administration by wistar rats. Open Neuropsychopharmacol J. 2009;2:77-85.

38. Guan Y, et al. GABAergic actions mediate opposite ethanol effects on dopaminergic neurons in the anterior and posterior ventral tegmental area. JPharmacol Exp Ther. 2012;341(1):33-42.
39. Buczynski MW, et al. Diacylglycerol lipase disinhibits VTA dopamine neurons during chronic nicotine exposure. Proc Natl Acad Sci U S A. 2016;113(4):1086-1091.

40. Melis M, et al. Enhanced endocannabinoid-mediated modulation of rostromedial tegmental nucleus drive onto dopamine neurons in Sardinian alcohol-preferring rats. J Neurosci. 2014;34(38):12716-12724.

41. Riegel AC, Lupica CR. Independent presynaptic and postsynaptic mechanisms regulate endocannabinoid signaling at multiple synapses in the ventral tegmental area. J Neurosci. 2004;24(49):11070-11078.

42. Melis $\mathrm{M}$ et al. Electrophysiological properties of dopamine neurons in the ventral tegmental area of Sardinian alcohol-preferring rats. Psychopharmacology (Berl). 2009;201(4):471-481.

43. Gianessi CA et al. Endocannabinoid contributions to alcohol habits and motivation: relevance to treatment. Addict Biol. 2020;25(3):e12768.

44. Morales I, et al. Effects of naltrexone on alcohol, sucrose, and saccharin binge-like drinking in C57BL/6J mice: a study with a multiple bottle choice procedure. Behav Pharmacol. 2020;31(2\&3):256-271

45. Navarro M, et al. Bupropion, alone and in combination with naltrexone, blunts binge-like ethanol drinking and intake following chronic intermittent access to ethanol in male C57BL/6J mice. Alcohol Clin Exp Res. 2019;43(5):783-790.

46. Crabbe JC, et al. High Drinking in the Dark (HDID) mice are sensitive to the effects of some clinically relevant drugs to reduce binge-like drinking. Pharmacol Biochem Behav. 2017;160:55-62.

47. Ho AMC, et al. Combined effects of acamprosate and escitalopram on ethanol consumption in mice. Alcohol Clin Exp Res. 2016;40 (7):1531-1539.

48. Cavener VS, et al. Inhibition of diacylglycerol lipase impairs fear extinction in mice. Front Neurosci. 2018;12:479.

49. Jenniches I, et al. Anxiety, stress, and fear response in mice with reduced endocannabinoid levels. Biol Psychiatry. 2016;79(10):858-868.

50. Hartley ND, et al. 2-arachidonoylglycerol signaling impairs short-term fear extinction. Transl Psychiatry. 2016;6(3):e749.

51. Lominac KD, et al. Behavioral and neurochemical interactions between Group $1 \mathrm{mGluR}$ antagonists and ethanol: potential insight into their anti-addictive properties. Drug Alcohol Depend. 2006;85(2):142-156.

52. Cozzoli DK, et al. The effect of mGluR5 antagonism during binge drinking on subsequent ethanol intake in C57BL/6J mice: sex- and age-induced differences. Alcohol Clin Exp Res. 2014;38(3):730-738.

53. Kumar J, et al. Effects of the mGluR5 antagonist MPEP on ethanol withdrawal induced anxiety-like syndrome in rats. Behav Brain Funct. 2013;9:43.

54. Lee KM, et al. mGlu5-dependent modulation of anxiety during early withdrawal from binge-drinking in adult and adolescent male mice. Drug Alcohol Depend. 2018;184:1-11.

55. Kasten CR, et al. Metabotropic glutamate receptor subtype 5 in alcohol-induced negative affect. 
Brain Sci. 2019;9(8):183.

56. Ceccarini J, et al. Recovery of decreased metabotropic glutamate receptor 5 availability in abstinent alcohol-dependent patients. J Nucl Med. 2020;61(2):256-262.

57. Kasten CR, et al. Adolescent alcohol exposure produces sex differences in negative affect-like behavior and group I mGluR BNST plasticity. Neu- ropsychopharmacology. 2020;45(8):1306-1315.

58. Grueter BA, et al. Extracellular-signal regulated kinase 1-dependent metabotropic glutamate receptor 5-induced long-term depression in the bed nucleus of the stria terminalis is disrupted by cocaine administration. J Neurosci. 2006;26(12):3210-3219.

59. Healey JC, et al. Chronic ethanol exposure leads to divergent control of dopaminergic synapses in distinct target regions. Alcohol. 2008;42(3):179-190.

60. Morgan AJ, et al. Detection of cyclooxygenase-2-derived oxygenation products of the endogenous cannabinoid 2-arachidonoylglycerol in mouse brain. ACS Chem Neurosci. 2018;9(7):1552-1559. 